\title{
Bendamustine in the treatment of non-Hodgkin's lymphomas
}

This article was published in the following Dove Press journal:

OncoTargets and Therapy

3 December 2009

Number of times this article has been viewed

\section{Fredrick Hagemeister ${ }^{1}$ George Manoukian²}

'Department of Lymphoma/Myeloma, The University of Texas M.D. Anderson Cancer Center Houston, TX, USA; ${ }^{2}$ Department of Internal Medicine, The University of Texas Health Science Center, Houston, TX, USA
Correspondence: Fredrick Hagemeister UT M.D. Anderson Cancer Center, Dept. of Lymphoma/Myeloma, I5I5 Holcombe Boulevard, Unit 429, Houston, TX 77030, USA

Tel +l 7|37454245

Fax +I 7137945656

Email fhagemei@mdanderson.org
Purpose: To review available data using bendamustine alone and in combination with other chemotherapeutic agents in treatment of patients with non-Hodgkin's lymphomas.

Methods: Internet database searches and literature review.

Results: Bendamustine was approved in March 2008 by the United States Food and Drug Administration for the treatment of patients with chronic lymphocytic leukemia. Many trials have been performed over the last decade using bendamustine not only as monotherapy, but also in combination with other agents including rituximab, vincristine, mitoxantrone, fludarabine, and other agents as therapy for patients with relapsed non-Hodgkin's lymphomas, and recently was approved for use in therapy of patients with relapsed indolent lymphomas considered refractory to rituximab therapy. As monotherapy, bendamustine induces good responses with only minor side effects. In combination with other agents, efficacy improves, especially when given in combination with rituximab. The drug has also been studied in combination with rituximab as initial therapy for indolent lymphomas, and has excellent activity with less toxicity than R-CHOP (rituximab - cyclophosphamide, hydroxydaunorubicin [Adriamycin], Oncovin [vincristine], and prednisone/prednisolone).

Conclusion: Overall, bendamustine has demonstrated promising results as therapy for nonHodgkin's lymphomas and should be included in the armamentarium of agents used to treat relapsed indolent non-Hodgkin's lymphomas and may prove valuable as initial therapy for these diseases. Further studies are being conducted to demonstrate the efficacy of this drug in combination with other agents.

Keywords: bendamustine, non-Hodgkin's lymphomas, relapsed lymphoma

\section{Indolent non-Hodgkin's lymphomas: Problems in choosing therapy}

Non-Hodgkin's lymphomas (NHL) are the most frequently occurring hematologic malignancies in the United States (US), responsible for approximately 19,000 deaths per year, with about 66,000 new cases in 2008. Since the 1970s, the incidence of NHLs has nearly doubled, increasing about $3 \%-4 \%$ per year. ${ }^{1}$ Even though some of this increase may be related to an increase in the incidence of acquired immunodeficiency syndrome (AIDS), for the most part, the main reason for this rise is unknown. Excluding cutaneous malignancies, NHLs are the fifth leading cause of new cancers in US, and the ninth leading cause of cancer death in males and seventh in females. Prior to the development of rituximab 1-, 5-, and 10-year survival rates were approximately $79 \%, 63 \%$, and $51 \%$, respectively. ${ }^{2}$ Indolent B-cell lymphomas account for approximately $30 \%-40 \%$ of the NHLs. After an initial response to a variety of

submit your manuscript | www.dovepress.con 
medications, most patients develop relapse, and response to second-line therapy is often incomplete, with shorter lasting remissions following subsequent treatments. Tolerance to relapse therapy is often poor, and resistance to standard agents develops in most; therefore, newer agents associated with good response rates and with low toxicity profiles are needed.

\section{Mechanism of action of bendamustine}

\section{Pharmacodynamics}

Bendamustine consists of three distinct structural groups: a purine analog-like benzimidazole ring, an alkylating agent group, and a water soluble alkane carboxylic chain. Bendamustine is categorized as an alkylating agent, causing intra- and interstrand DNA cross-links, leading to disruption of DNA matrix function and DNA synthesis. ${ }^{3}$ The drug causes more cross-linking then other alkylators; this seems to be more durable and more difficult to repair than those induced by carmustine and cyclophosphamide. ${ }^{4}$ However, unlike other alkylating agents, bendamustine also causes activation of DNA damage via stress response and apoptosis pathways, inhibition of mitotic checkpoints, and induction of mitotic catastrophe. ${ }^{5}$ As compared to equitoxic concentrations of phosphoramide mustard and chlorambucil, bendamustine more strongly induces genes responsible for apoptosis. Bendamustine also leads to phosphorylation of Ser-15 and increases protein expression of Bax, both of which play a role in p53-induced apoptosis, and inhibits mitotic check points by inhibiting several mitosis-related genes. ${ }^{5,6}$

\section{Pharmacokinetics}

The drug is available as an intravenous formulation, infused over 30-60 minutes; peak plasma concentration $\left(C_{\max }\right)$ occurs at the end of the infusion. ${ }^{7}$ It has been studied in treatment of hematologic malignancies at doses of 50-60 mg/m² daily for three or five days every four weeks, or $100-120 \mathrm{mg} / \mathrm{m}^{2}$ daily for two days, every three weeks, whereas in solid tumors, doses were higher (120-150 mg/m² daily, every four weeks). Bendamustine is highly protein bound, mainly to albumin, and protein binding is not affected by older age $(>70$ years) or by low serum albumin levels; however, unbound bendamustine is the only form that is active. ${ }^{8,9}$ After a single dose of $120 \mathrm{mg} / \mathrm{m}^{2}$, the intermediate terminal half life of the parent compound is approximately 40 minutes, with initial elimination via the kidneys, although the drug has not been studied in patients with renal failure. ${ }^{6,8,10}$ Bendamustine also undergoes extensive first pass hepatic metabolism, primarily by hydrolysis, via the cytochrome p450 system, and it should be given cautiously to patients with significant hepatic dysfunction. ${ }^{7,11}$ The major metabolite is $\beta$-hydroxybendamustine, which is also cytotoxic but does not contribute significantly to the drug's activity, since concentrations of this metabolite are well below that of the parent compound. ${ }^{7}$ Phase II conjugation with glutathione also plays an important role in metabolism; preclinical radiolabeled bendamustine studies showed that approximately $90 \%$ of the drug administered was recovered primarily in the feces. ${ }^{7,9}$

\section{Clinical trials of bendamustine for therapy of relapsed NHL Monotherapy: Phase II trials (Table I)}

The types of patients in trials of bendamustine for treatment of NHL have varied, probably accounting for some of the differences in results among studies. Overall response rates (OR) have varied from $73 \%$ to $100 \%$; complete response rates (CR) have varied from $11 \%$ to $34 \%$, with partial response rates (PR) from $43 \%$ to $62 \%$. The median duration of response (DR) in these trials also varied from 6.7 to 39 months. Many of the phase I/II studies of bendamustine in the treatment of NHL were reported in the mid 1990s; investigators in Germany also studied the drug as monotherapy in treatment of chronic lymphocytic leukemia (CLL) in a randomized trial compared with chlorambucil. Because of the results of this trial, the drug was approved by the American Food and Drug Administration (FDA) for therapy of CLL in the US. Investigators in the US have also studied the drug as monotherapy for patients with relapsed indolent lymphoma; results of these two rigorous studies have confirmed that bendamustine is an active agent in treatment of indolent lymphomas in relapse, and can be administered with the advantage of good tolerance, with the only major side effects being modest myelosuppression at the doses suggested in these trials, and minimal non-hematologic toxicity, making it a potential drug to combine with other agents in therapy of lymphomas. Moreover, many of these trials were conducted in patients known to have disease considered resistant to rituximab, a population with disease often difficult to treat.

\section{European trials of bendamustine for relapsed $\mathrm{NHL}$}

Although bendamustine was invented in the 1960s, studies documenting its true effectiveness did not begin in earnest until the 1990s. In 2001, Heider and Niederle reported on a phase II trial of bendamustine in therapy of 58 patients with 
Table I Phase II trials of bendamustine as monotherapy for patients with relapsed or refractory NHL

\begin{tabular}{|c|c|c|c|c|c|c|}
\hline Author & $\begin{array}{l}\text { Dose } \\
\left(\mathrm{mg} / \mathrm{m}^{2}\right)\end{array}$ & $\begin{array}{l}\text { Schedule of } \\
\text { drug delivery }\end{array}$ & $\begin{array}{l}\text { Number of } \\
\text { patients }\end{array}$ & $\%$ OR & $\% \mathbf{C R}$ & $\begin{array}{l}\text { Remission duration } \\
\text { (median, months) }\end{array}$ \\
\hline Heider and Niederle ${ }^{12}$ & 120 & $\begin{array}{l}\text { Days I, } 2 \\
\text { Q three weeks }\end{array}$ & 52 & 73 & 11 & 16 \\
\hline Bremer $^{7}$ & 60 & $\begin{array}{l}\text { Days } 1-5 \\
\text { Q four weeks }\end{array}$ & 62 & 82 & 15 & 39 \\
\hline Weidmann ${ }^{17}$ & 120 & $\begin{array}{l}\text { Days } 1,2 \\
\text { Q three weeks }\end{array}$ & 18 & 44 & 17 & NR \\
\hline Friedberg ${ }^{26}$ & 120 & $\begin{array}{l}\text { Days I,2 } \\
\text { Q three weeks }\end{array}$ & 74 & 77 & 34 & 6.7 \\
\hline $\mathrm{Kahl}^{27}$ & 120 & $\begin{array}{l}\text { Days I,2 } \\
\text { Q three weeks }\end{array}$ & 100 & 76 & 33 & 9.2 \\
\hline
\end{tabular}

Abbreviations: $\mathrm{CR}$, complete response; $\mathrm{NHL}$, non-Hodgkin's lymphoma; OR, overall response; $\mathrm{Q}$, .

relapsed NHL, using $120 \mathrm{mg} / \mathrm{m}^{2}$ daily, given days 1 and 2 on a three-week cycle. ${ }^{12}$ Fifty-two of these patients were evaluable and all had at least one prior therapy. The median number of treatments prior to bendamustine was one (range 1-6), but the median interval from the end of prior therapy to bendamustine was only three months. The median number of bendamustine cycles was six (range 1-11), and the median follow-up time was 24 months (range 1-67). Results were impressive when compared to those for other single agents in a comparably treated population in prior studies: the OR was $73 \%$, with $11 \% \mathrm{CR}$, and $62 \%$ PR. Purine analogs (cladribine, pentostatin, fludarabine) have provided $30 \%-70 \%$ response rates in similar populations; CR was uncommon in these studies, and occasionally associated with opportunistic infections, not reported by Heider and Niederle. ${ }^{13-16}$ Weidmann and colleagues also reported results of a phase II trial with bendamustine for patients with relapsed aggressive lymphomas using $120 \mathrm{mg} / \mathrm{m}^{2}$ daily on days 1 and 2, every three weeks. ${ }^{17}$ Most of these patients were judged as ineligible for aggressive combinations of therapy or were known to have drug-resistant disease. Twelve of them had diffuse large cell lymphoma (DLCL) and three had human immunodeficiency virus-positive serologies. Of the 18 evaluable patients on this study, $44 \%(8 / 18)$ had a response: $17 \%$ with $\mathrm{CR}$ and $28 \%$ with PR. Only four of the patients had disease refractory to previous treatment and all four had a response to bendamustine, two with CR and two with PR. The median DR was not reported in this trial; however, response durations in the three patients in CR were 6, 8+, and 27+ months. In this study, grades $3-4$ hematologic toxicities occurred in up to $13 \%$ of the patients, and the drug was discontinued in two due to prolonged thrombocytopenia and leukopenia. No grade 4 nonhematologic adverse events were reported, and grade 3 nonhematologic toxicities occurred in less than
$10 \%$ of the patients. In this population, investigators have reported response rates of $30 \%$ or less, and CRs are rare in patients with refractory disease. ${ }^{18-24}$ Both of these studies documented that the drug is an active single agent in treatment of a wide variety of NHLs, and encouraged exploration of its use in other studies, as a single agent and in combinations with other drugs.

In another phase II trial, using a lower dose of bendamustine, but a longer duration of treatment, Bremer and colleagues administered bendamustine at $60 \mathrm{mg} / \mathrm{m}^{2}$ daily for five days at four-week intervals in 62 patients with relapsed lymphoproliferative disorders, including mostly patients with myelomas or immunocytomas (also known as lymphoplasmacytic lymphomas [LPL]). ${ }^{7}$ The median number of treatments was two (range 1-10) and most of these patients had previously received chlorambucil, with or without prednisone, or melphalan, both alkylating agents. They reported an OR of $82 \%$ with a CR of $15 \%$, PR of $67 \%$, and a median DR of 39 months: for the 62 patients with NHL, the OR was $82 \%$, with a median DR of 39 months. Although the authors concluded that this dose and schedule may have produced longer DRs than those employed by Heider and Niederle and others, histologies were not comparable, and it is difficult to conclude which schedule might best exploit the value of this drug. Furthermore, a five-day schedule is perhaps less convenient than a two-day schedule, and without additional studies, it is difficult to reach any conclusion about this trial. Nonetheless, this study suggests the value of this drug in management of yet two more histologies, myeloma and LPL.

\section{North American trials of bendamustine for relapsed $\mathrm{NHL}$}

Rituximab refractoriness may become an important determinant of response for relapsed lymphomas, not only for 
indolent, but also aggressive lymphomas. ${ }^{22,25}$ In 2007 , Friedberg and colleagues reported results of a phase II trial using bendamustine, $120 \mathrm{mg} / \mathrm{m}^{2}$ daily on days 1 and 2 , given every three weeks, as therapy for 76 evaluable patients with rituximab-refractory indolent or transformed NHL. ${ }^{26}$ In this multicenter study, rituximab refractoriness was defined as no response or progression within six months of completing rituximab therapy, alone or combined with other drugs. Histologies included indolent phenotypes (follicular [FL], $61 \%$; small lymphocytic [SLL], 16\%; marginal zone [MZL], $3 \%$; other, 14\%) and transformed lymphomas. The median number of prior therapies was two (range 1-5) and prior treatments included single-agent rituximab (76\%), CHOP-like or CVP (cyclophosphamide, prednisone, vincristine) therapy with or without rituximab (82\%), autologous stem cell transplant (ASCT, 8\%), and radioimmunotherapy (RIT, 12\%). In the 74 patients evaluable for response, the OR was $77 \%$, with a CR of $34 \%$ and PR of $43 \%$. The median DR was 6.7 months for all responders; for those with disease considered refractory to prior alkylator therapy, it was $61 \%$, with a CR of $30 \%$. The median progression-free survival (PFS) rate was 7.1 months; it was 4.2 months for patients with transformed disease and 8.3 for those with indolent lymphomas. In this trial, the median number of cycles of therapy per patient was five (range 1-9). Primary side effects included reversible myelosuppression, with grades 3-4 neutropenia (54\%), febrile neutropenia (7\%), and thrombocytopenia $(25 \%)$. Three patients who had previously received extensive therapy have developed myelodysplastic syndrome or leukemia. This study was important because it was the first trial evaluating a chemotherapeutic agent in a group of patients with disease considered refractory to rituximab, and encouraged the pivotal trial in the US that enabled approval of bendamustine in therapy of relapsed indolent lymphomas.

The pivotal study leading to approval by the FDA of bendamustine for therapy of relapsed indolent lymphomas in the US was a multicenter, single-armed trial, conducted by Kahl and colleagues in 100 patients; rituximab resistance was defined as no response or progression within six months of 1) receiving the first dose of a full course of rituximab induction, 2) completion of rituximab maintenance therapy or progression before the next scheduled rituximab dose, or 3 ) completion of a full course of the combination of rituximab and chemotherapy. ${ }^{27}$ Patients could have received additional systemic therapy after the qualifying rituximab regimen. The treatment program was similar to that used in the trial described by Friedberg and colleagues, with administration of $120 \mathrm{mg} / \mathrm{m}^{2}$ of bendamustine daily on days 1 and 2, every three weeks; the plan was to administer only six cycles, although patients could receive a maximum of eight cycles if clinical benefit was observed. Histologies included FL (62\%), SLL (21\%), MZL (16\%), and LPL (1\%). In this population, the median number of prior rituximab-containing regimens was two (range 1-6), and the number of prior chemotherapy regimens administered was two (range 0-6). Twenty-four percent had previously received RIT, and 36\% were considered to have disease considered refractory to their last chemotherapy regimen. The FLIPI index was also studied in the patients with FL prior to relapse therapy. ${ }^{28}$ Response rates were high, as in other trials, ranging from $71 \%-100 \%$ for various histologies. Complete response rates by histologies were: FL (20\%), SLL (5\%), nodal MZL (11\%), and extranodal MZL (43\%). The OR for patients known to have disease considered refractory to their last chemotherapy regimen (that is, no $\mathrm{CR}$ or $\mathrm{PR}$ ) was $64 \%$, including $6 \% \mathrm{CR}$, and $88 \%$ for those known to have chemosensitive disease, with $30 \% \mathrm{CR}$. In this trial, the FLIPI score at the time of relapse did not predict which patients would respond or enter CR: the CR rate for those FLIPI scores of 2 was $27 \%$, and for those with FLIPI scores of 3 or more, it was $23 \%$. The median DR for all patients was 6.3 months, and the median PFS was 7.5 months. Grades 3-4 hematologic toxicities were the only major side effects, and similar to those noted in the study reported by Friedberg and colleagues. The use of growth factors was not specified in the study: $15 \%$ had grade 3 infections, and $6 \%$ grade 4 . Based on the results of this trial, bendamustine was approved for therapy of indolent lymphomas in relapse following documentation of rituximab-based resistance. More importantly, results of this study and that of Friedberg and colleagues paved the way for development of other protocols in the US, many of which are still ongoing.

\section{Bendamustine in combination with other agents for relapsed NHL: Phase II trials (Table 2)}

As with most drugs used in therapy of patients with B-cell lymphomas, rituximab is often the first drug to be added to its administration schema, in hopes that the combination of rituximab plus the drug in question will benefit the patient population by delivering a higher response rate, especially $\mathrm{CR}$, and a longer DR. Few trials have addressed this possibility in a randomized fashion for relapsed disease using single agent therapy, although investigators have documented this principle in treatment of patients with combination chemotherapy. ${ }^{29,30}$ Therefore, when bendamustine was found to be an active agent, it was combined with rituximab and other agents in phase II trials. In these studies, most of 
Table 2 Phase I/II trials of bendamustine in combination with other agents in therapy of relapsed and refractory NHL

\begin{tabular}{|c|c|c|c|c|c|}
\hline Authors & $\begin{array}{l}\text { Other agents used } \\
\text { in trials }\end{array}$ & $\begin{array}{l}\text { Number of } \\
\text { patients }\end{array}$ & $\%$ OR & $\% \mathbf{C R}$ & $\begin{array}{l}\text { Duration of response } \\
\text { (median, months) }\end{array}$ \\
\hline Rummel $^{31,32}$ & Rituximab & 166 & 90 & 60 & PFS 30 months \\
\hline Lakner ${ }^{35}$ & Rituximab & 46 & 85 & 43 & PFS 12 months \\
\hline Mohren ${ }^{36}$ & Rituximab & 10 & 70 & 40 & NR \\
\hline Robinson ${ }^{37}$ & Rituximab & 66 & 92 & 54 & 21 \\
\hline Koenigsman ${ }^{38}$ & Fludarabine & 29 & 77 & 45 & 14 \\
\hline Weide ${ }^{42}$ & Mitoxantrone, rituximab & 55 & 89 & 35 & PFS 19 months \\
\hline Matous $^{47}$ & Bortezomib, rituximab & 16 & 80 & 53 & NR \\
\hline
\end{tabular}

Abbreviations: CR, complete response; NHL, non-Hodgkin's lymphoma; NR, no response; OR, overall response; PFS, progression-free survival.

which were conducted in Europe prior to the approval of bendamustine by the FDA in the US, and some of which were published only in German, it is not clear that the addition of other chemotherapy agents to the combination of rituximab and bendamustine (BR) provided any major benefit over that expected with BR alone, suggesting that further studies would be needed to determine the best combination of drugs to use for patients with relapsed NHL when treated with BR.

\section{Bendamustine and rituximab for relapsed $\mathrm{NHL}$}

Rummel and colleagues performed in vitro studies demonstrating that rituximab enhances the activity of bendamustine in a synergistic fashion. ${ }^{31}$ They found that the doses of bendamustine required to induce apoptosis in DOHH-2 cells was halved when rituximab was added to the media, with similar effects demonstrated in studies with freshly isolated B-CLL cells. Based on this observation, they treated a group of patients with relapsed or refractory low-grade or mantle cell lymphomas using the BR combination. ${ }^{32}$ In this trial, 63 eligible patients, $30 \%$ of whom had disease considered refractory to their last chemotherapy, received the BR regimen, using bendamustine $90 \mathrm{mg} / \mathrm{m}^{2}$ daily on days 1 and 2, with the standard dose of rituximab, $375 \mathrm{mg} / \mathrm{m}^{2}$ on day 1 , given every four weeks. Patients received a maximum of four cycles. Histologies included FL (38\%), MCL (25\%), LPL (27\%), and MZL (10\%). The OR was 90\%, with a CR or $60 \%$, and a median PFS of 24 months. This PFS was significantly longer that that reported following the patients' treatments prior to $\mathrm{BR}$ (nine months). OR and CR rates in this trial for various histologies were: FL, $96 \%$ and $71 \%$; LPL (also designated small lymphocytic), 100\% and 53\%; MCL, $75 \%$ and $50 \%$; and MZL, $83 \%$ and $67 \%$, respectively. Interestingly, the median PFS was 18 months for those with MCL, whereas the median PFS for patients with
FL and LPL had not been reached, with a median follow-up of 20 months. In an updated analysis of the data, the median duration of survival had not been reached, with a median PFS of 30 months. ${ }^{33}$ Grades 3-4 toxicities were assessed per cycle: neutropenia occurred in 16\% of cycles, thrombocytopenia in $3 \%$, and anemia in only $1 \%$. Most importantly, no growth factors were administered during the course of this trial. This group of investigators is also conducting an ongoing trial of BR in the treatment of patients aged over 75 years. In a recent analysis, $30 \%$ of the patients enrolled had FL and $18 \%$ MCL, and $36 \%$ of these patients had received prior therapy. ${ }^{34}$ In this report regarding 33 evaluable patients, the OR was $91 \%$, with $30 \%$ CR. One patient died during the first cycle of therapy of neutropenic fever; otherwise, the safety profile of this regimen was similar to that reported for younger patients, suggesting that it might be an ideal regimen for the older patient, especially one with co-morbid health issues that might preclude more intensive therapy.

Lakner and colleagues have also conducted a prospective trial of BR in therapy of relapsed or refractory indolent and MCL, using $90 \mathrm{mg} / \mathrm{m}^{2}$ of bendamustine on days 1 and 2 , and rituximab at the standard dose of $375 \mathrm{mg} / \mathrm{m}^{2}$ on day $1 .{ }^{35} \mathrm{The}$ median age was 69 , and histologies included FL (25 patients), MCL (10), LPL (8), and MZL (3). The OR was $85 \%$, with $43 \% \mathrm{CR}$, and the median event-free survival (EFS) result was nine months (range, 0-39), with a PFS of 12 months. These results are very similar to those reported by Rummel and colleagues, and provide further evidence of the effectiveness of this regimen; however, more importantly, the combination is not associated with cardiac, pulmonary, renal, or hepatic toxicity, an outcome similar to that expected with these drugs when used as single agents.

Mohren and colleagues have evaluated the safety and efficacy of another method of administration of bendamustine and rituximab in treatment of 10 evaluable patients 
with relapsed or refractory aggressive NHLs. ${ }^{36}$ All patients enrolled had previously received at least one treatment that included an anthracycline. Patients received bendamustine $60 \mathrm{mg} / \mathrm{m}^{2}$ on days 1,8 , and 15 every four weeks, and rituximab $375 \mathrm{mg} / \mathrm{m}^{2}$ on days $1,8,15$, and 22 during the first cycle only. In this study, three had a PR, four had CR, and three of those entering CR are still in remission at six, 18, and 33 months. Further evaluation of this treatment schedule would be of significant interest.

\section{North American trial}

In a phase II multicenter study of BR, using similar doses and schedules of drugs to Rummel and colleagues, investigators treated 66 patients with relapsed indolent and mantle cell lymphomas: none had disease considered to be refractory to rituximab. ${ }^{37}$ The median age was 60 years ( $40-84$ years), and the median number of cycles was six (2-7 cycles). The OR was $92 \%$, with 54\% CR. Overall response and CR rates for 54 with indolent histologies were $93 \%$ and $54 \%$; corresponding results for the 12 with MCL were $92 \%$ and $59 \%$. More importantly, there were only minor differences between the results for those who had previously received rituximab and those who had not, including ORs of $87 \%$ and $100 \%$, and CRs of $49 \%$ and $62 \%$, respectively. The median followup time on this trial was 20 months, with a median DR of 21 months for all patients, and 21 and 19 months for those with indolent and MCL, respectively. In this study, febrile neutropenia was rare $(7 \%)$ despite the fact that grades 3-4 neutropenia occurred in $37 \%$, a finding common to most trials that include bendamustine as a single agent or in combination with rituximab. Importantly, growth factor or blood product support was administered during only $9 \%$ of the cycles, and only $12 \%$ of the patients received granulocyte growth factors, increasingly used as the number of treatment cycles increased. Five deaths occurred in this trial, causes including disease progression (three patients), compartment syndrome (one), and toxic epidermal necrolysis (one): the last of these occurred in the setting of multiple drug administrations, including allopurinol, a drug associated with this type of complication of therapy. Regardless, reports of this complication prompted a warning in the package insert regarding the safety of bendamustine.

\section{Bendamustine and other chemotherapy agents for relapsed or refractory $\mathrm{NHL}$} Bendamustine and fludarabine

In a phase I/II trial, Koenigsman and colleagues reported results of therapy for 29 patients using bendamustine and fludarabine. ${ }^{38}$ This combination was chosen because the investigators hypothesized that the alkylating activity of bendamustine could be enhanced when combined with a purine analog; incorporation of the analog might prevent normal DNA synthesis, thereby increasing the alkylating agent's activity, an observation noted with in vitro studies of apoptosis using the two drugs in combination. ${ }^{39-41}$ Two dose levels of bendamustine were employed, $30 \mathrm{mg} / \mathrm{m}^{2}$ (nine patients) and $40 \mathrm{mg} / \mathrm{m}^{2}$ (20 patients), and all received fludarabine at $30 \mathrm{mg} / \mathrm{m}^{2}$. Each drug was administered on days 1,2 , and 3 , and plans were to administer the combination every four weeks for a maximum of six cycles. Fourteen were considered to have chemotherapy-refractory disease, and 14 had previously received anti-CD20 therapy; six had undergone prior ASCT. The toxicity of the regimen was mainly hematologic, and no growth factors were used in this trial. Nine of 19 evaluable patients had grade 3 , and five had grade 4 hematotoxicities, with grades 3-4 thrombocytopenia in $2 \%$ of the cycles and grades 3-4 neutropenia in $25 \%$. Eighteen percent of the patients in the phase I portion of this trial experienced febrile neutropenia, and one in the phase II portion died of neutropenic sepsis. There was also a tendency for patients to experience cumulative cytotoxicity with the administration of more treatment cycles. Of 22 patients evaluable for response, the OR was 77\%, with $45 \% \mathrm{CR}$, and $70 \%$ of all patients required a maximum of four cycles for best response; 10 patients were unable to receive 6 cycles as intended because of myelotoxicity. The authors recommended that this combination, using $30 \mathrm{mg} / \mathrm{m}^{2}$ of each drug, was effective in treatment of indolent and MCL, but warned that the fewer number of cycles given to achieve response, the better the combination would be tolerated.

\section{Bendamustine, mitoxantrone, and rituximab}

Weide and co-workers have studied this combination in therapy of patients with relapsed or refractory indolent or MCL or CLL, in a phase I/II single center study, and have reported results of a phase II multicenter trial. ${ }^{42-44}$ In the former study, 20 patients were treated within the phase I aspect of the trial, and 34 received the doses determined for the phase II portion, using bendamustine $90 \mathrm{mg} / \mathrm{m}^{2}$ daily for patients with NHL and $80 \mathrm{mg} / \mathrm{m}^{2}$ for those with CLL, days 1 and 2, mitoxantrone $10 \mathrm{mg} / \mathrm{m}^{2}$ on day 1 , and rituximab $375 \mathrm{mg} / \mathrm{m}^{2}$ on days $8,15,22$, and 29. ${ }^{42,43}$ From days 36 on, patients received only bendamustine and mitoxantrone every 4 weeks as with the first cycle of therapy, for a maximum of six cycles. Histologies included indolent 
NHL (26 patients), transformed NHL (six), B-cell CLL (21), and prolymphocytic leukemia (one). Fludarabine had been previously administered to $12 \%$ of those with indolent NHL, $27 \%$ of those with leukemias, and $17 \%$ of the patients with transformed NHL: only five patients had previously received rituximab. The OR for all patients on this trial was $96 \%$, with a CR of $41 \%$. The CR rate for FL was $57 \%$; for transformed NHL it was $33 \%$, and was $50 \%$ and $75 \%$ for those with LPL and MCL/MZL, respectively. The median time to progression (TTP) was 7.5 months in those with transformed NHL, but had not been reached in the indolent NHL group after a median follow-up of 27 months. This last group had a median time to next treatment result of only 6.5 months with the therapy given to them immediately prior to BMR.

In the subsequent phase II, multicenter trial, Weide and colleagues treated 57 patients with relapsed or refractory indolent and MCL, using BMR differently than that originally described (bendamustine $90 \mathrm{mg} / \mathrm{m}^{2}$ days 1 and 2, mitoxantrone $10 \mathrm{mg} / \mathrm{m}^{2}$ on day 1 , rituximab $375 \mathrm{mg} / \mathrm{m}^{2}$ on day 8 ), with cycles repeated every four weeks, for a total of four cycles. ${ }^{44}$ Overall, 39\% had previously received rituximab, although only two were considered to have disease that was resistant to that drug. Of the 55 evaluable patients, the OR was $89 \%$, with $35 \%$ CR, and the median PFS 19 months, with a median overall survival (OS) of 33 months. OR and CR rates for those with MCL (18 patients) and FL (27 patients) were $78 \%$ and $33 \%$, and $92 \%$ and $50 \%$, respectively. Median PFS and OS results were 21 and 31 months, and 17 months and not reached, respectively, with a median follow-up time of 27 months (1-43 months). Interestingly, results for those who had previously received rituximab were similar to those for patients who had not. In this trial, as would be expected, grades 3-4 hematologic toxicities included granulocytopenia in $46 \%$, and thrombocytopenia in $16 \%$, with grade 3 nonhematologic toxicities in less than $6 \%$ for each type and none that were grade 4 in severity. Although the response rates for this combination may seem to be excellent, it is unclear what mitoxantrone adds to this combination, and only a randomized trial of BMR and BR would answer the question as to which might produce better results for these patients.

\section{Bendamustine and bortezomib for relapsed or refractory $\mathrm{NHL}$}

Moosmann and colleagues have recently reported results of a phase I/II trial of nine patients with relapsed (five patients) or refractory (four patients) indolent or MCL. ${ }^{44}$ In this group of heavily pretreated patients, seven had already received rituximab, two prior bortezomib, two ASCT, and two RIT.
Treatment consisted of bendamustine $60 \mathrm{mg} / \mathrm{m}^{2}$ (phase I) escalated to $80 \mathrm{mg} / \mathrm{m}^{2}$ (phase II) on days 1,8 , and 15 and bortezomib $1.6 \mathrm{mg} / \mathrm{m}^{2}$ on days $1,8,15$, and 22; a similar trial has suggested that, when combined with rituximab, higher doses of bortezomib given weekly may be as effective as lower doses given twice weekly, with less toxicity. ${ }^{46}$ Doselimiting toxicities with this combination included diarrhea, fatigue, and thrombocytopenia; however, there were no serious infectious or neuropathic serious adverse events in this study. The phase II portion of this trial is ongoing.

\section{Bendamustine, bortezomib, and rituximab for relapsed or refractory follicular lymphomas}

In this single-armed multicenter trial, also known as the VERTICAL study, Matous and colleagues have treated 16 patients with relapsed or refractory $\mathrm{FL}$, all of whom had previously undergone ASCT for relapse, and had previously received rituximab, $63 \%$ of whom had disease considered refractory to the antibody. ${ }^{47}$ As opposed to the patient selection in the study by Moosmann and colleagues, no patient enrolled on this study could have previously received bortezomib or bendamustine. This phase I/II study was designed to find the optimal, maximally tolerated dose of bendamustine that could be administered to patients receiving bortezomib $1.6 \mathrm{mg} / \mathrm{m}^{2}$ on days $1,8,15$, and 22 every 35 days, with the bendamustine administered on days 1 and 2, another difference between this trial and the study by Moosmann and colleagues. The maximum tolerated dose of bendamustine was primarily defined by duration of hematologic toxicity, and, based on this study, was $90 \mathrm{mg} / \mathrm{m}^{2}$. Rituximab $375 \mathrm{mg} / \mathrm{m}^{2}$ was administered during the first cycle on days $1,8,15$, and 22 , and in subsequent cycles was given only on day 1 . The regimen was associated with grades 3-4 neutropenia and thrombocytopenia in $25 \%$ and $12 \%$, respectively, and the median number of cycles administered was five. Peripheral neuropathy occurred in $44 \%$ of the patients, with a median time to onset of 12 weeks; however, it resolved in all who had grades 1-2 neuropathy, and persisted in only one who had grade 3 neuropathy and discontinued therapy. Overall, $80 \%$ of the patients had a response, with $53 \% \mathrm{CR}$, and response rates were not clearly related to the dose of bendamustine administered. Responses occurred within the first three cycles of therapy in most patients: six of the 12 responders had disease considered refractory to their last rituximab-containing treatment. This is an ongoing trial; the regimen has not been compared to the $\mathrm{BR}$ regimen, and only a randomized study can determine the value of bortezomib in this combination. 


\section{Clinical trials of bendamustine} in combination with other agents as initial therapy for patients with NHL (Table 3)

\section{Bendamustine, vincristine, and prednisone for therapy of indolent and $\mathrm{MCL}$}

In a phase III trial, patients with stage III-IV indolent or MCL received therapy that was randomized to bendamustine, vincristine, and prednisone (BOP, 82 patients) versus cyclophosphamide, vincristine, and prednisone (COP, 80 patients). ${ }^{48}$ A maximum of eight cycles of therapy was planned on each arm of this trial. Overall, $86 \%$ had stage IV disease; $50 \%$ had FL, 27\% had MCL, and 23\% had LPL. Requirements for enrollment included presence of B symptoms, thrombocytopenia or anemia, rapid tumor growth, or bulky disease: exclusions included stage III with less than five nodal regions of disease, with all nodes less than $5 \mathrm{~cm}$ in diameter. Doses initially included vincristine $2 \mathrm{mg}$ on day 1 and prednisone $100 \mathrm{mg} / \mathrm{m}^{2}$ daily on days $1-5$ on both arms of this trial. The dose of cyclophosphamide on the COP regimen was $400 \mathrm{mg} / \mathrm{m}^{2}$ daily intravenously on days 1-5 for all cycles of therapy; however, the original protocol dose of bendamustine was $70 \mathrm{mg} / \mathrm{m}^{2}$ daily on days $1-5$. Due to significantly more thrombocytopenia with BOP than COP after enrollment of 25 patients onto this trial, the dose of bendamustine was reduced to $60 \mathrm{mg} / \mathrm{m}^{2}$ daily on days $1-5$ in all subsequent cycles of BOP. To further complicate matters, interferon maintenance was to be administered to all patients who entered CR or PR on this trial; of 115 eligible patients, only 28 actually received this drug. Regardless of the drawbacks to the design and performance of this trial, BOP was as effective as COP at inducing remissions: OR and $\mathrm{CR}$ rates were $66 \%$ and $22 \%$ (BOP) versus $76 \%$ and $20 \%$ (COP), respectively ( $P=0.1$ ). Of note, a patient who might be considered as having a $\mathrm{CRu}$ in US trials might be considered as having a PR in this study. The five-year TTP result for responders to BOP was 59\% compared to $46 \%$ for those responders who received COP $(P=0.037)$. EFS results were affected by the administration of interferon: responders who received BOP or COP had similar results, but those who responded to BOP had longer time to treatment failure than did those who responded to COP. Five-year overall survival rates were similar on the two arms of this trial, although they were better when patients received interferon maintenance, and were lower for patients with MCL (46\%) than they were for those with FL or LPL (66\% and 74\%, respectively). Grades 3-4 toxicities were uncommon, except for neutropenia, which occurred more frequently in patients receiving COP.

\section{Bendamustine and rituximab as therapy for previously untreated indolent and $\mathrm{MCL}$} Rummel and colleagues have conducted a trial that has completed accrual for patients with previously untreated indolent and MCL, randomizing treatments between $\mathrm{BR}$, as originally described by this group in its original trial of patients with relapsed disease, and R-CHOP (rituximab - cyclophosphamide, doxorubicin, vincristine, prednisone), using standard doses, given every three weeks. ${ }^{49,50}$ Of the first 463 patients enrolled on this trial, histologies were equally distributed between both arms: these included FL (52\%), MCL (19\%), LPL/SLL (14\%), and MZL (13\%). In their report of results for the first 315 patients on this study, BR was not only as effective as R$\mathrm{CHOP}$ at inducing remissions, with similar $\mathrm{OR}$ and $\mathrm{CR}$ rates for the two arms of this trial, but BR was more tolerable than R-CHOP. In this phase III, noninferiority study, the OR and CR rates for 162 patients on the BR arm were 93\% and $47 \%$ compared with $93 \%$ and $42 \%$ for the 149 patients receiving R-CHOP. Response rates were similar for both treatments for all histologies: OR rates for patients with FL,

Table 3 Phase III randomized trials of bendamustine in combination with other agents for previously untreated NHL

\begin{tabular}{|c|c|c|c|c|c|}
\hline Author & $\begin{array}{l}\text { Combinations used } \\
\text { in trials }\end{array}$ & $\begin{array}{l}\text { Number of } \\
\text { patients }\end{array}$ & $\%$ OR & $\% \mathbf{C R}$ & Duration of response \\
\hline \multirow[t]{3}{*}{ Herold $^{48}$} & Bendamustine, & 82 & 66 & 22 & Five-year TTP \\
\hline & vincristine, & vs & vs & vs & $59 \%$ vs $46 \%$ for \\
\hline & prednisone vs COP & 80 & 76 & 20 & responders \\
\hline \multirow[t]{3}{*}{ Rummel $^{49,50}$} & Bendamustine, & 162 vs 149 & 93 & 47 & Median PFS not reached \\
\hline & rituximab vs & & vs & vs & vs 39 months for all \\
\hline & $\mathrm{R}-\mathrm{CHOP}$ & & 93 & 42 & patients \\
\hline
\end{tabular}

Abbreviations: COP, cyclophosphamide, vincristine, prednisone; $\mathrm{CR}$, complete response; NHL, non-Hodgkin's lymphoma; OR, overall response; $\mathrm{PFS}$, progression-free survival; R-CHOP, rituximab - cyclophosphamide, hydroxydaunorubicin [Adriamycin], Oncovin [vincristine], and prednisone/prednisolone; TTP, time to progression. 
LPL/SLL, MCL, and MZL were $96 \%, 100 \%, 88 \%$, and $83 \%$, respectively; corresponding OR results for those receiving R-CHOP were $93 \%, 86 \%, 96 \%$, and $100 \%$. CR rates for these histologies using BR were $51 \%, 45 \%, 42 \%$, and $42 \%$; those for patients receiving R-CHOP were 43\%, 24\%, 41\%, and $56 \%$. In a follow-up of this trial, response rates were still similar to those originally reported by this group. ${ }^{48}$ With a median follow-up of 27 months on both arms of the trial, results were not inferior for those patients receiving BR (232 patients) compared to those for patients receiving R-CHOP (224 patients): progression or relapse has been observed in 63 treated with BR and in 89 treated with R-CHOP. The median PFS for those on the R-CHOP arm is 39 months; for those who received BR, it has not yet been reached. Grades 3-4 neutropenia were observed in 38\% and 14\% of the R-CHOP and BR arms, respectively, and $21 \%$ of those receiving $\mathrm{R}-\mathrm{CHOP}$ received granulocyte colony-stimulating factor (G-CSF) compared with only 5\% of those receiving BR. Infectious complications also occurred more frequently in those treated with R-CHOP (31\% for BR compared with $41 \%$ with R-CHOP, including all grades). Of course, bendamustine is not associated with significant cardiac, renal, or hepatic toxicity, alopecia does not occur, and neuropathy is rare: all of these are toxicities that investigators have reported with R-CHOP. Final results of this trial are awaited: patients with some histologies may have superior PFS results with one or the other regimen, once data is sorted according to various histologic subtypes. Results of this trial have also encouraged the development of an international confirmatory trial: this study may lead to the approval by the FDA of bendamustine in combination with rituximab as initial therapy for indolent and mantle cell lymphomas.

\section{Bendamustine and rituximab as initial therapy for aggressive B-cell NHLs}

Following their finding that bendamustine has activity in therapy of patients with recurrent aggressive NHLs, Weidmann and colleagues conducted a trial in which they evaluated the efficacy and safety of BR as initial therapy for patients over the age of 80 years who were not considered eligible for R-CHOP therapy. ${ }^{51}$ The dose of bendamustine was escalated in this trial compared to the $\mathrm{BR}$ regimen described by Rummel and colleagues. ${ }^{32}$ Those with stage I-II disease received four cycles of BR every 21 days, using bendamustine $120 \mathrm{mg} / \mathrm{m}^{2}$ daily on days 2 and 3 , with the standard dose of rituximab on day 1 , followed by involved field radiotherapy. Those with stage III-IV disease received the same regimen for a total of six cycles, followed by two extra doses of rituximab. Twelve of the 14 patients enrolled on this trial had DLCL; eight had stage I-II disease. Six (55\%) of the 11 evaluable patients achieved CR, and two (18\%) a PR. The median PFS was 14.4 months, and the estimated two-year OS was 53\%. Grades 3-4 neutropenia occurred in only $18 \%$ of the administered cycles and only $4 \%$ developed grade 3 thrombocytopenia.

\section{Ongoing trials with bendamustine: An old drug becomes a new drug}

Currently, there are a number of trials which are attempting to determine the best way to utilize this drug, invented so long ago. Multiple investigators have reported variations of the dose administration described by Rummel and colleagues, using $90 \mathrm{mg} / \mathrm{m}^{2}$ in combination with rituximab for relapsed disease, daily on days 1 and 2; in the original study reported by Heider and Niederle and in the North American trials of single-agent therapy, the dose was $120 \mathrm{mg} / \mathrm{m}^{2}$, and in the trial for B-cell CLL, it was $100 \mathrm{mg} / \mathrm{m}^{2}$. At the present time, it is also unclear what the best schedule might be for the drug, since most of the single-agent trials have used dosing every three weeks, and in the BR trials reported by Rummel and colleagues, the drugs are given every four weeks, for which the dose of bendamustine is significantly lower than that approved by the FDA as a single agent. In combinations that include myelotoxic agents, including fludarabine, and anthracyclines, the maximal dose may be $90 \mathrm{mg} / \mathrm{m}^{2}$, as suggested by some trials, although allowable dose modifications and routine use of growth factors may prevent some of the associated reported neutropenic episodes and infections encountered with multiple agent chemotherapy. However, when administered in combination with rituximab, or any other antibody, the appropriate dose may well be $90 \mathrm{mg} / \mathrm{m}^{2}$, as suggested by Rummel and colleagues, even though higher doses of bendamustine might be just as safe as the lower dose administered in their trial. Besides safety, other reasons to consider adoption of the BR schedule reported by Rummel and associates relate to ease of administration, given over two days, rather than at lower doses over five days, or weekly, as in some trials. In order to demonstrate that alternate regimens provide improved results, randomized trials would be needed, although it may be difficult to conduct such studies, given the number of new drugs available to the treating physician.

Besides tolerability issues, BR may be as good as regimens that employ bendamustine and rituximab plus some other chemotherapy agent, including fludarabine, anthracyclines, and spindle cell toxins. To date, there has been no randomized study documenting that any regimen is 
better than the BR combination, and comparisons between different studies is difficult, due to wide variability in the diseases studied and prior treatments administered to these populations. Part of this problem stems from the effectiveness of rituximab: almost all regimens, whether single-agent or combination therapies, provide better results for patients with B-cell NHL when administered with rituximab. Many of the studies described above have administered rituximab using schemas that are different than those described by Rummel and colleagues: one dose prior to each cycle of chemotherapy has become a standard of care, although other methods may provide better results. It is possible that BOP may be as good as COP, as reported by Herold and colleagues; however, this study remains primarily of historical interest, especially now that rituximab is widely available. Only randomized studies can determine the effectiveness of any regimen compared to another.

Finally, novel agents for therapy of lymphomas are on the horizon: these include protein inhibitors, proteasome inhibitors, anti-CD20 antibodies, immunomodulators, and antibodies directed against other targets on the cell surface. All of these might be agents that could be combined with bendamustine, and the VBR trial (bendamustine, bortezomib, rituximab) is a good example of newer studies. Hopefully, this "old but new" agent will eventually find its proper place among the list of drugs available to treat patients with lymphoproliferative diseases in our armamentarium, with its good tolerability and ease of administration.

\section{Acknowledgments}

The authors would like to thank Thao Phan for her secretarial assistance in preparation of this manuscript.

\section{References}

1. Cancer Facts and Figures 2008. Atlanta, GA: American Cancer Society; 2008. Available from: http://www.cancer.org/downloads/ STT/2008CAFFfinalsecured.pdf. Accessed June 23, 2009.

2. Brockman B. Therapy of the recurrence of malignant lymphoma. ZArztl Fortbild (Jena). 1992;86:843-846.

3. Hartmann M, Zimmer CH. Investigation of cross-link formation in DNA by alkylating cytostatic IMET 3106, 3393 and 3943. Biochem Biophys Acta. 1972;287:386-389.

4. Strumberg D, Harstrick A, Doll K, et al. Bendamustine hydrochloride activity against doxorubicine-resistant human breast cancer cell lines. Anticancer Drugs. 1996;7:415-421.

5. Leoni LM, Bailey B, Reifert J, et al. Bendamustine (Treanda) displays a distinct pattern of cytotoxicity and unique mechanistic features compared with other alkylating agents. Clin Cancer Res. 2008;14:309-317.

6. Weide R. Bendamustine HCL for the treatment of relapsed indolent non-Hodgkin's lymphoma. Ther Clin Risk Manage. 2008;4:727-732.

7. Bremer K. High rates of long-lasting remissions after 5-day bendamustine chemotherapy cycles in pre-treated low-grade non-Hodgkin's lymphomas. J Cancer Res Clin Oncol. 2002;128:603-609.
8. Owen JS, Melhem M, D'Andrea D, Darwish M. Population pharmacokinetics of bendamustine and metabolites in patients with indolent non-Hodgkin lymphoma. Clin Pharmacol Ther. 2008; 83:(Suppl 1):S54-S55. (Abstract P11-41).

9. Balfour JA, Goa KL. Bendamustine. Drugs. 2001;61(5):631-640.

10. Matthias M, Preiss R, Sohr R, et al. Pharmacokinetics of bendamustine in patients with malignant tumors [abstract]. Proc Am Soc Clin Oncol. 1995; 14:1476.

11. Preiss R, Sohr R, Matthias M, et al. The pharmacokinetics of bendamustine (Cytostasan) in humans. Pharmazie. 1985;40:782-784.

12. Heider A, Niederle N. Efficacy and toxicity of bendamustine in patients with relapsed low-grade non-Hodgkin's lymphoma. Anticancer Drugs. 2001;12:725-729.

13. Tulpule A, Schiller G, Harvey-Buchanan LA, et al. Cladribine in the treatment of advanced relapsed or refractory low and intermediate grade non-Hodgkin's lymphoma. Cancer. 1998;83:2370-2376.

14. McLaughlin P, Robertson LE, Keating MJ. Fludarabine phosphate in lymphoma: an important new therapeutic agent. In: Cabanillas F, Rodriguez MA, editor. Advances in Lymphoma Research. Boston, MA: Kluwer Academic; 1997. p. 3-14.

15. Dearden CE, Matutes E, Catovsky D. Clinical overview of pentostatin (Nipent) use in lymphoid malignancies. Semin Oncol. 2000;27(2 Supp15): 22-26.

16. Cabanillas F, Horning S, Kaminski M, Champlin R. Managing indolent lymphomas in relapse: Working our way through a plethora of options. Hematology Am Soc Hematol Educ Program. 2000:166-179.

17. Weidmann E, Kim SZ, Rost A, et al. Bendamustine is effective in relapsed or refractory aggressive non-Hodgkin's lymphoma. Ann Oncol. 2002;13:1285-1289.

18. Younes A, Preti HA, Hagemeister F, et al. Paclitaxel plus topotecan treatment for patients with relapsed or refractory aggressive non-Hodgkin's lymphoma. Ann Oncol. 2001;12:923-927.

19. Savage DG, Rule SAJ, Tighe M, et al. Gemcitabine for relapsed or resistant lymphoma. Ann Oncol. 2000;11:595-597.

20. Coiffer B, Haioun C, Ketterer N, et al. Rituximab (anti-Cd20 monoclonal antibody) for the treatment of patients with relapsing or refractory aggressive lymphoma: A multicenter phase II study. Blood. 1998;96:1927-1932.

21. Wiernik P, Lossos I, Tuscano J, et al. Lenalidomide monotherapy in relapsed or refractory aggressive non-Hodgkin's lymphoma. $J$ Clin Oncol. 2008;26:4952-4957.

22. Czuczman M, Vose J, Zinzani P, et al. Confirmation of the efficacy and safety of lenalidomide oral monotherapy in patients with relapsed or refractory diffuse large B-cell lymphoma: Results of an international study (NHL-003). Blood (ASH Annual Meeting Abstracts). 2008:268.

23. Johnston P, Ansell S, Colgan J, et al. Phase II trial of the oral mTOR inhibitor everolimus (RAD001) for patients with relapsed or refractory lymphoma. 2007 ASCO Annual Meeting Proceedings - Post meeting edition; J Clin Oncol. 2007;25(18S):8055.

24. Witzig T, Geyer S, Ghobrial I, et al. Phase II trial of single-agent temsirolimus (CCI-779) for relapsed mantle cell lymphoma. J Clin Oncol. 2005;23:5347-5356.

25. Coiffier B, Feugier P, Sebban C, et al. Long term results of the GELA study, R-CHOP vs CHOP in elderly patients with diffuse large B-cell lymphoma. Blood (ASH Annual Meeting Abstracts). 2004;104:1383.

26. Freidberg JW, Cohen P, Chen L, et al. Bendamustine in patients with rituximab-refractory indolent and transformed non-Hodgkin's lymphoma: Results from a phase II multicenter, single-agent study. $J$ Clin Oncol. 2008;26(2):204-210. Erratum in: J Clin Oncol. 2008;10(26):1911.

27. Kahl BS, Bartlett N, Leonard J, et al. Bendamustine is safe and effective in patients with rituximab-refractory indolent B-cell NHL. Proceedings of the 49th Annual Meeting of the American Society of Hematology; 2007 Dec 4-8; Atlanta, GA. Blood. 2007:1351.

28. Solal-Céligny P, Roy P, Colombat P, et al. Follicular lymphoma international prognostic index. Blood. 2004;104:1258-1265. 
29. Forstpointner R, Dreyling M, Repp R, et al; The addition of rituximab to a combination of fludarabine, cyclophosphamide, mitoxantrone (FCM) significantly increases the response rate and prolongs survival as compared with FCM alone in patients with relapsed and refractory follicular and mantle cell lymphomas: Results of a prospective randomized study of the German Low-Grade Lymphoma Study Group. Blood. 2004;104:3064-3071.

30. van Oers M, Klasa R, Marcus R, et al. Rituximab maintenance improves clinical outcome of relapsed/resistant follicular non-Hodgkin lymphoma in patients both with and without rituximab during induction: Results of a prospective randomized phase 3 intergroup trial. Blood. 2006;108:3295-3301.

31. Rummel MJ, Chow KU, Hoelzer D, Mitrou PS, Weidmann E. In vitro studies with bendamustine: Enhanced activity in combination with rituximab. Semin Oncol. 2006;29:12-14.

32. Rummel MJ, Al-Batran SE, Kim SZ, et al. Bendamustine plus rituximab is effective and has a favorable toxicity profile in the treatment of mantle cell and low-grade non-Hodgkin's lymphoma. J Clin Oncol. 2005;23:3383-3389.

33. Rummel MJ, Welslau AM, Kofahl-Krause D, et al. Bendamustine and rituximab (BR) are effective in the treatment of relapsed or refractory indolent and mantle cell lymphomas: Long-term follow-up of a phase II study. Chicago, IL: Proceedings from the 43rd Annual Meeting of the American Society of Clinical Oncology; 2007 Jun 2-5. Blood. 8034.

34. Rummel MJ, Heine K, Bodenstein H, et al. Efficacy and safety of bendamustine and rituximab in the treatment of indolent and mantle cell lymphomas in older patients. Chicago, IL: Proceedings from the 44th Annual Meeting of the American Society of Clinical Oncology. J Clin Oncol. 2008;Suppl:8572.

35. Lakner V, Eschenburg H, Aldaoud A, et al; Bendamustine and rituximab in the treatment of relapsed CD20+ non-Hodgkin lymphoma - a phase II study of the East German Study Group of Haematology and Oncology. Lugano, Switzerland: Proceedings from the 10th International Conference on Malignant Lymphoma. 2008 June 4-7.

36. Mohren M, Herold M, Scheinpflug K, et al. Bendamustine/ Mabthera in patients with relapsed or refractory aggressive CD20+ B-cell lymphoma. Lugano, Switzerland: Proceedings from the 10th International Conference on Malignant Lymphoma; 2008 June 4-7.

37. Robinson KS, Williams ME, van der Jagt RH, et al. Phase II multicenter study of bendamustine plus rituximab in patients with relapsed indolent B-cell and mantle cell non-Hodgkin's lymphoma. J Clin Oncol. 2008;26(27):4473-4479.

38. Koenigsmann M, Knauf W, Herold M, et al. Fludarabine and bendamustine in refractory and relapsed indolent lymphoma - a multicenter phase I/II trial of the East German society of hematology and oncology (OSHO). Leuk Lymphoma. 2004;45:1821-1827.

39. Ghandi V. Metabolism and mechanisms of action of bendamustine: Rationales for combination therapies. Semin Oncol. 2002;29(Suppl 13): 4-11.

40. Schwãnen $C$, Hecker T, Hũbinger $G$, et al. In vitro evaluation of bendamustine induced apoptosis in B-chronic lymphocytic leukemia. Leukemia. 2002;16:2096-2105.
41. Chow KU, Boehrer S, Geduldig K, et al. In vitro induction of apoptosis of neoplastic cells in low-grade non-Hodgkin's lymphomas using combinations of established cytotoxic drugs with bendamustine. Haematologica. 2001;86:485-493.

42. Weide R, Heymanns J, Gores A, Koppler H. Bendamustine, mitoxantrone and rituximab (BMR): A new effective regimen for refractory or relapsed indolent lymphomas. Leuk Lymphoma. 2002;43:327-331.

43. Weide R, Pandorf A, Heymanns J, et al. Bendamustine/mitoxantrone/ rituximab (BMR): A very effective, well tolerated outpatient chemoimmunotherapy for relapsed and refractory CD20-positive indolent malignancies. Final results of a pilot study. Leuk Lymphoma. 2004;45:2445-2449

44. Weide R, Hess G, Koeppler H, et al; High anti-lymphoma activity of bendamustine/mitoxantrone/rituximab (BMR) in rituximab pretreated relapsed or refractory indolent lymphomas. A multicenter phase II study of the German low grade lymphoma study group (GLSG). Leuk Lymphoma. 2007;48:1299-1306.

45. Moosmann PR, Heizmann M, Kotrubczik N, et al. Weekly treatment with a combination of bendamustine and bortezomib in relapsed or refractory indolent NHL: A single-center phase I/II study. San Francisco, CA: Proceedings from the 50th Annual American Society of Hematology Meeting. 2008 Dec 6-9; Blood. 1574.

46. de Vos S, Dakhil S, McLaughlin P, et al. Phase 2 study of bortezomib weekly or twice weekly plus rituximab in patients with follicular lymphoma (FL) or marginal zone (MZL) lymphoma: Final results. Orlando, FL: Proceedings from the 48th Annual American Society of Hematology Meeting. 2006 Dec 8-12; Blood. 2006:694.

47. Matous J, Letzer J, Rosen P, et al. Bortezomib, bendamustine, and rituximab in patients with relapsed or refractory follicular lymphoma: Dose finding results of the VERTICAL trial. Orlando, FL: Proceedings from the 45th Annual Meeting of the American Society of Clinical Oncology. 2009 May 29-June 2; J Clin Oncol. 8550.

48. Herold M, Schulze A, Neiderweiser D, et al. Bendamustine, vincristine and prednisone (BOP) versus cyclophosphamide, vincristine and prednisone (COP) in advanced indolent non-Hodgkin's lymphoma and mantle cell lymphoma: Results of a randomized phase III trial (OSHO \# 19). J Cancer Res Clin Oncol. 2006;132:105-112.

49. Rummel MJ, von Gruenhagon U, Niederle N, et al. Bendamustine plus rituximab versus CHOP-R in the first-line treatment of patients with indolent and mantle cell lymphomas-first interim results of a randomized phase III study of the StiL (Study Group Indolent Lymphomas, Germany). Atlanta, GA: Proceedings from the 49th Annual American Society of Hematology Meeting. 2007 Dec 8-11; Blood. 385.

50. Rummel MJ, von Gruenhagon U, Niederle N, et al. Bendamustine plus rituximab versus CHOP plus rituximab in the first-line treatment of patients with follicular, indolent, and mantle cell lymphomas: Second interim analysis of a randomized phase III study of the Study Group Indolent Lymphomas (StiL). San Francisco, CA: Proceedings from the 50th Annual American Society of Hematology. 2008 Dec 6-8; Blood. 2596

51. Weidmann E, Fauth F, Atmaca A, et al. First-line treatment with rituximab and bendamustine in old patients ( 80 years and older) with aggressive B-cell lymphomas - an interim analysis of an ongoing phase II study. Berlin, Germany: Proceedings from the 14th Congress of the European Hematology Association. 2009 June 4-7.
OncoTargets and Therapy

\section{Publish your work in this journal}

OncoTargets and Therapy is an international, peer-reviewed, open access journal focusing on the pathological basis of all cancers, potential targets for therapy and treatment protocols employed to improve the management of cancer patients. The journal also focuses on the impact of management programs and new therapeutic agents and protocols on

\section{Dovepress}

patient perspectives such as quality of life, adherence and satisfaction The manuscript management system is completely online and includes a very quick and fair peer-review system, which is all easy to use. Visit http://www.dovepress.com/testimonials.php to read real quotes from published authors. 